\title{
Découvrir la forêt - CH Waldwochen - Vivere il bosco La forêt, nature et entreprise, objet de formation globale
}

\section{Introduction}

Découvrir la forêt - $\mathrm{CH}$ Waldwochen - Vivere il bosco est un programme national d'éducation et de formation en milieu forestier. Il occupe depuis 1985 une place particulière dans ce qu'on appelle "l'éducation à l'environnement", et s'engage aussi dans la formation professionnelle des forestiers dans le domaine de l'information et de la communication. Créée en pleine crise du «dépérissement des forêts» pour soutenir une approche positive de la forêt par les enseignants, cette action s'est en effet étendue depuis à d'autres publics: forestiers, animateurs de groupes de jeunes, familles, entreprises. Des projets se déroulent en Suisse romande et alémanique ainsi qu'au Tessin et occupent à temps partiel une quinzaine d'enseignant(e)s, biologistes et ingénieurs forestiers. Ce programme fonctionne sur une base privée, tout en étant soutenu de façon déterminante par la Confédération et de nombreux cantons.

\section{De la frustration au plaisir:}

\section{pour ne plus agir sans savoir ou savoir sans agir?}

Beaucoup de défenseurs de l'environnement se plaignent de la contradiction qu'ils pensent constater entre "ce que tout le monde sait» sur les problèmes de l'environnement et "l'absence de changement de comportement» qu'on observe dans notre société. Ils demandent aux spécialistes du comportement ou aux éducateurs d'expliquer ce phénomène, respectivement de mieux éduquer les jeunes générations.

Avant de présenter la réponse, pratique, d'un programme comme Découvrir la forêt, il me semble nécessaire de poser les questions suivantes:

- Est-ce vrai que nos concitoyens ou concitoyennes «savent», mais n' «agissent» pas?

- S'ils savent, par exemple, ce que disent les journaux sur le trou d'ozone, est-ce qu'alors ils le croient pour autant? Vraiment?

- Et s'ils croient vraiment que le problème du trou d'ozone existe, est-ce qu'ils ont la possibilité d'agir? Laquelle?

- Et s'ils connaissent des possibilités d'agir, est-ce qu'ils se sentent concernés, responsable de faire, par exemple, un premier pas?

Ces questions peuvent être aussi posées en rapport avec le dépérissement des forêts, le gaspillage d'énergie, l'es- clavage des enfants en Amérique ou en Asie, les échanges nord-sud et bien d'autres thèmes. A l'inverse, le lecteur, la lectrice, peut se demander, dans un domaine ou son engagement personnel est évident, d'où est venue sa motivation? Est-ce vraiment une information ou une campagne publicitaire sur un probleme de notre société qui a joué l'effet décisif? S'il s'agissait d'un effet déclencheur, par quoi était constitué le terreau où la graine de l'action a germé?

Le programme appelé Découvrir la forêt ( $\mathrm{CH}$ Waldwochen, Vivere il bosco) apporte une réponse pratique en se basant sur une hypothèse: ce que l'on aime ou respecte, on est prêt à le protéger. La question est donc: peut-on faire aimer la nature, la forêt, les êtres humains? S'il est certain qu'on ne peut forcer quiconque à aimer ou à respecter, on peut créer des occasions de rencontre. Et si la rencontre est positive, motivante, créative, conviviale, alors l'occasion est donnée, en toute liberté, d'aimer et de respecter. Et si c'est le cas, toujours selon notre hypothèse de travail, alors le savoir et l'action n'évolueront pas isolément.

\section{Exemple du dépérissement des forêts}

Le dépérissement des forêts a, surtout lorsque les médias en parlaient encore régulièrement, insécurisé aussi les éducateurs. Comment traiter le thème de la forêt sans être écrasé par le négatif et, dans un réflexe compréhensible de refoulement, risquer d'éloigner encore davantage les jeunes de la nature? Une démarche d'approche positive de la forêt est née de cette question. Au-delà des messages angoissants sur la "mort des forêts", à la longue usants et abstraits, des pédagogues ont proposé de remplacer l'angoisse et la frustration par l'action et le plaisir. La forêt, même malade, est un lieu inépuisable de découvertes et d'expériences. A travers le plaisir de découvrir, un attachement s'opère avec la forêt et la nature. Les discussions sur les problèmes écologiques n'en sont pas moins présentes et importantes, mais elles sont accompagnées d'expériences personnelles positives et par la possibilité d'agir.

Philippe Domont, Ingénieur forestier EPFZ, Zschokkestraße 18, 8037 Zürich 


\section{Le choix de la forêt}

Le choix de la forêt comme objet et lieu d'éducation s'est révélé particulièrement fructueux pour des raisons pratiques et thématiques. La forêt peut en effet être considérée comme une micro-société où les problèmes de notre société et leur solution peuvent être étudiés:

- L'importance économique, sociale et culturelle de la forêt et de l'arbre dans notre société est énorme: bois, protection, loisirs, «idée de nature», diversité biologique, paysage, traditions populaires, mythologies, art.

- Le dépérissement des forêts reste un probleme pédagogique non résolu pour les éducateurs: symbole des destructions effectives ou potentielles de l'homme dans la nature, il est très difficile de faire un bilan répondant au souci ou à la curiosité des enfants et des adultes sur ce sujet. Les informations scientifiques contiennent beaucoup de points d'interrogations, même si l'urgence du problème est maintenant relativisée.

- L'entreprise forestière - que constitue chaque commune avec son personnel forestier - est un exemple vivant d'interdépendance entre société et nature. On peut l'observer facilement et recevoir l'appui des forestiers.

- Les aspects fondamentaux des activités du service forestier peuvent servir d'exemple de gestion durable des ressources naturelles aux autres branches de l'économie (maintien de la biomasse, réflexion à long terme, pondération entre éléments économiques, sociaux, écologiques et culturels).

- La gestion de la forêt et le fonctionnement de l'écosystème sont très mal connus du public et des éducateurs, ces derniers ayant des difficultés à aborder ces thèmes dans leur travail.

- La forêt n'est jamais très loin du lieu d'habitation ou de travail, elle est toujours ouverte au public et offre partout une richesse naturelle étonnamment bien conservée (ou recréée depuis une centaine d'années) dans notre paysage civilisé.

- La forêt interpelle des couches profondes de la personne: en même temps qu'elle offre sa protection (on s'y sent protégé, à l'abri), la forêt fait aussi peur, par exemple la nuit; on a peur de s'y faire attaquer ou de s'y perdre. Elle est ainsi un lieu symbolique de découverte de soi, de croissance, face à ses aspirations et à ses peurs.

\section{Démarche pédagogique}

La découverte de la forêt se fait donc dans le but de créer une relation de plaisir, de curiosité ou encore de respect envers la forêt, dans l'hypothèse que cette base positive soit intégrée par l'adulte dans les décisions privées ou professionnelles qu'il est amené à prendre. Le projet est conçu à long terme et s'adresse avant tout aux jeunes, que l'on cherche à atteindre par l'intermédiaire de leurs éducateurs: enseignants, parents, animateurs, personnages de références (par exemple les forestiers).
La démarche pédagogique proposée doit permettre aux éducateurs de proposer une approche positive et diversifiée de la forêt, de son importance socio-économique comme de sa diversité biologique, de sa beauté comme des atteintes auxquelles elle est soumise. Les démarches où interviennent des groupes d'éducateurs en forêt s'inspirent des méthodes modernes de pédagogie des adultes, où la participation des apprenants est considérée comme une condition de l'apprentissage. Cela dit, nous n'avons pas besoin de réinventer la pédagogie. Beaucoup se trouve résumé dans le principe «tête, cœur, main» cher à Pestalozzi, appliqué à la forêt:

- la relation cognitive avec le milieu: une approche des espèces végétales et animales, aux liens écologiques, mais aussi aux interventions de l'homme dans le cadre de l'économie forestière

- la relation affective, plus difficile à réaliser et à décrire, où un vécu lié à la forêt doit avoir lieu: discussion au sein d'un groupe, rencontre avec des "personnages de la forêt" comme le forestier ou le chasseur, réflexion, observations captivantes de jour ou de nuit, aventures, histoires ou contes

- la relation physique avec le milieu: jeu, travaux forestiers, marche, bivouac.

\section{Moyens utilisés}

L'essentiel du travail d'éducation en forêt se répartit en:

- cours de perfectionnement pour divers publics de pédagogues, animateurs ou vulgarisateurs (durée de 2 à 5 jours): le contenu des cours touche à la découverte de la forêt sous les divers aspects cités tout en cherchant à montrer les possibilités de retransmettre ces découvertes à des jeunes

- documents didactiques: brochures et articles dans la presse professionnelle sur des thèmes touchant la forêt et les activités pédagogiques qui peuvent s'y dérouler

- actions: elles regroupent des cours et documents didactiques sur un thème donné (exemple: «Une nuit à la belle étoile" ou "Forêt magique - magie de la forêt»)

- conseils, soutien pédagogique: de nombreuses demandes émanant des enseignants et des groupes de jeunes peuvent être satisfaites par l'envoi de matériel et par l'assistance sur place lors d'une action ou journée forestière

- travail d'information et de coordination visant à initier une collaboration entre les diverses organisations forestières, pédagogiques ou écologiques s'intéressant à la forêt (exemple: coordination nationale de la Journée internationale de la forêt).

\section{Des expériences avec cinq publics-cibles}

\section{Les écoles: les enseignants ont-ils encore peur du méchant loup?}

On ne parle d'éducation à l'environnement que depuis un peu plus d'une dizaine d'années. Dans son rapport «Edu- 
cation à l'environnement dans les écoles suisses» (1988), la Conférence suisse des Directeurs cantonaux de l'instruction publique présente la nécessité de ce nouveau thème par «les progrès techniques ainsi que la conduite irresponsable de l'homme face à la nature (qui) mettent notre planète en danger». Selon ce même rapport, l'éducation à l'environnement intègre l'interdisciplinarité (biologie, histoire, chimie, sociologie, droit, etc.) et l'étude de situations concrètes (étude de cas, contact direct avec les autorités, spécialistes, etc.). Elle veut non seulement transmettre des connaissances, mais aussi permettre d'agir. N'est-ce pas là une déclaration des plus progressistes?

La tâche d'"enseigner la forêt», thème inclus dans les programmes scolaires, n'est pas facile pour les enseignants: ils avouent presque tous avoir des difficultés à aborder une matière qu'ils ressentent comme compliquée (voir les résultats de l'enquête de Lausanne ci-dessous). Pour que la forêt ne reste pas une "jungle impénétrable" pour les enseignants - et donc pour la jeunesse - un soutien didactique est donc approprié. Par le perfectionnement des enseignants, surtout au niveau primaire, le projet transmet des méthodes didactiques modernes facilitant l'intégration de la forêt dans l'apprentissage des élèves. Il s'agit actuellement de:

- méthodes de pédagogie active pour la découverte de la flore (plus spécialement des arbres), de la faune, des interactions dans l'écosystème, de la sylviculture, des bois (essences, bois dans la vie quotidienne)

- contact avec les professionnels de la forêt et du bois

- documentation.

\section{Les forestiers: le dépérissement des forêts a catalysé les activités d'information}

Le dépérissement des forêts a surpris l'administration, la recherche et les praticiens forestiers à une époque où presque personne ne s'intéressait de près ni à leur travail, ni à la forêt. De leur côté, au fonds des bois comme au fonds des laboratoires, les forestiers n'avaient jusqu'alors pas manifesté le besoin d'informer ouvertement et activement le public. (C'est en 1983 seulement que les premiers services d'information forestiers ont vu le jour au niveau national.) Avec le dépérissement des forêts et l'intégration de la forêt dans la politique de protection de l'environnement, les forestiers ont dû s'exprimer beaucoup plus souvent sur leur travail, sur la forêt, donner des avis techniques et politiques sous les feux de l'actualité. Les difficultés habituelles liées à la communication, renforcées par le manque d'habitude, se sont alors faites sentir:

- Comment parler à des non-connaisseurs de la forêt, enfants ou adultes, expliquer des situations socio-économiques et écologiques complexes?

- Comment réagir de façon constructive face à la critique, garder le dialogue?

Les cours de perfectionnement ont donc pour objet l'organisation de la communication avec un groupe autour des themes forestiers complexes par nature, au carrefour de l'économie et de l'écologie. Les techniques de com- munication ne sont en effet pas encore intégrées dans les programmes de formation professionnelle. Même si, aujourd'hui, les feux de l'actualité se sont faits moins brûlants pour les forestiers, la nécessité de communiquer et le rôle du forestier dans l'éducation à l'environnement grandissent quand même d'année en année.

\section{Groupes de jeunes: \\ Perfectionnement des animateurs nécessaire}

Au contraire des écoles, les groupes de jeunes (scouts, cadets, colonies, etc.) se tiennent souvent dans la forêt. Mais cela ne signifie pas pour autant qu'ils découvrent ce milieu, considéré en général comme la coulisse d'autres activités. Les animateurs et moniteurs ne sont cependant pas préparés à une tâche de découverte différenciée du milieu et ont très peu de possibilités de se perfectionner. Pourtant, l'intérêt de réaliser une tâche d'éducation en faveur de l'environnement grandit dans les organisations de jeunes. Les demandes en conseils et en formation adressées à Découvrir la forêt augmentent. Nous trouvons important de jeter des ponts entre les jeunes et la nature durant leurs activités extrascolaires. Leur contribution potentielle au respect de la forêt et à un engagement plus efficace dans la société pour la gestion des ressources naturelles est non négligeable.

\section{Les familles: tourisme doux, loisirs actifs en forêt}

Le temps consacré aux loisirs augmente et, on peut l'espérer, aussi le besoin de vivre des expériences enrichissantes - et non polluantes. Le tourisme doux et les vacances actives semblent être en progression, d'après les observations des milieux touristiques. Des animateurs accompagnent des familles durant des «vacances forestières actives» d'un weekend ou d'une semaine. Des cours de formation pour animateurs spécialisés dans le milieu familial ont débuté. Un des aspects intéressants de cette démarche est la possibilité pour parents et enfants de s'engager en commun dans des activités de recherche, de découverte, de créativité en forêt.

\section{Les entreprises}

Il s'agit d'un projet dans sa phase initiale qui, s'adressant au personnel et aux apprentis des entreprises, leur offre des activités de découverte active de l'environnement forestier.

\section{La relation des enseignants avec la forêt: quelques résultats d'une enquête}

La forêt faisant partie des thèmes inscrits au programme scolaire, il a paru intéressant d'étudier l'intégration effective de ce theme par les enseignant(e)s et de faire ressortir d'éventuelles difficultés. En octobre 1991, Découvrir la forêt a mené une enquête représentative auprès de 246 
enseignants lausannois sur leur façon d'intégrer la forêt dans l'enseignement. Il va sans dire qu'il ne s'agissait pas de montrer quiconque du doigt, mais de saisir comment les enseignants pouvaient être secondés dans leur tâche. Les principaux thèmes des questions posés ont touchés

- la fréquence des visites en forêts par les classes

- les activités réalisées en forêt par les enseignants avec leur classe

- les difficultés que rencontrent les enseignants à traiter le thème de la forêt

- les souhaits des enseignants pour mieux pouvoir intégrer la forêt dans leurs cours.

\section{Voici quelques-unes des questions posées et quelques conclusions ${ }^{2}$}

a) Quelle est la fréquence de vos visites en forêts avec votre classe et combien de temps durent-ils?

Conclusion: environ la moitié des enseignants ne se rend que 0 à 2 fois par an en forêt avec sa classe, l'autre moitié s'y rendant 3 ou 4 fois ou plus. Les trois-quarts des séjours en forêts ne durent qu'une demi-journée (c'est-àdire en moyenne 2 heures), un quart une journée. La durée des séjours en forêt de loin la plus fréquente est d'une demi-journée. En relation avec la fréquence des visites, on peut déduire que la moitié des élèves ne passent qu'une demi-journée à une journée en forêt par année. Cela ne suffit pas, de loin, à établir une relation avec ce milieu.

\section{b) Désirez-vous changer la fréquence de vos visites en forêt?}

Conclusion: les trois-quarts des enseignants désirent se rendre plus souvent en forêt avec la classe, un quart à la même fréquence.

\section{c) Quelles activités réalisez-vous en forêt?}

\section{Conclusion:}

Les activités beaucoup citées sont: excursion, détente, connaissance des plantes et animaux, sport et jeux. Les activités plus ou moins souvent citées: activités manuelles, connaissance de l'environnement, dessin, géographie.

Les activités peu citées: contact avec un professionnel, travaux pratiques forestiers, histoire, langues, conte, littérature.

\section{d) Comment ma formation professionnelle m'a préparéa in- tégrer la forêt dans mon enseignement?}

Conclusion: $22 \%$ des enseignants ne se sentent "pas du tout» et $67 \%$ se sentent "un peu», $8 \%$ "suffisamment» et $3 \%$ «bien" préparé à enseigner le thème "forêt». Le perfectionnement ou l'accompagnement peut donc jouer un rôle important de soutien pour les enseignants.

e) Quels sont les éléments qui rendent l'enseignement du thème de la forêt difficile?
Conclusion: les éléments qui rendent l'enseignement du thème de la forêt difficile sont avant tout, d'après les enseignants:

- le manque de temps (programme chargé)

- le manque de documents didactiques (le manque de documents de base comme les livres est moins cité)

- le manque de connaissances sur la forêt.

Les résultats de l'enquête, dont certains sont rapportés ici, ont confirmé les expériences faites lors du perfectionnement ou de l'accompagnement des enseignants durant ces dernières années en Suisse. La somme de ces informations permet de tirer les conclusions suivantes:

1. La grande majorité des enseignants désire intégrer la forêt dans l'enseignement. Ils trouvent utile et important de s'y rendre avec leurs élèves.

2. Les enseignants connaissent mal la forêt, que ce soit au niveau écologique (arbres et autres plantes, faune, sol, facteurs climatiques, ou les interactions entre ces facteurs), socio-économique (travaux d'exploitation, de gestion, fonction de la forêt pour notre société) ou encore culturel (mythologie, contes, litterature).

3. Bien qu'ils apprécient la forêt, les enseignants hésitent à s'y rendre, car ils ne savent pas quoi y faire "pour l'école» et se sentent peu sécurisé dans ce milieu pédagogiquement inconnu. De plus, leurs programmes sont toujours plus chargés et ils ont tendance à écarter les contacts avec l'extérieur, contacts qui prennent du temps. Ce qui pourrait aider les enseignants:

- des méthodes de découverte collective d'un milieu inconnu

- la volonté de collaborer avec des milieux extérieurs à l'école

- un perfectionnement "généraliste»sur les aspects socio-économiques, écologiques et culturels de la forêt

- davantage de place pour ces démarches dans la formation pédagogique.

\section{Le développement durable à la porte de l'école}

Après le sommet de Rio, on a compris que la planète Terre peut et doit être gérée en commun. Le développement durable était dans toutes les bouches. Une des démarches qui concerne l'école et relevée pour son importance est celle de l'éducation. Si la réalisation planétaire du développement durable n'est pas pour demain, l'éducation doit l'intégrer dès aujourd'hui. Il existe déjà des exemples concrets, bien développés même sans être parfaits, qui montre le chemin de la gestion durable des ressources. La gestion des forêts, plus elle se rapproche de ce que les forestiers nomment une "sylviculture proche de la nature», en est un exemple. Elle est illustré ci-dessous par ses principes «traduits» en langage écologique, principes qui sont appliqués pour l'essentiel sur l'ensemble du territoire. Les aspects sont mis en opposition (de façon volontairement schématique) aux tendances de la société 
de consommation. La sylviculture proche de la nature permet de démontrer les comportements suivants:

\section{Etre conscient, étant individu, de n'être qu'un maillon dans une chaine qui le dépasse}

- intégrer son travail (soins, exploitations) dans celui des générations précédentes (un vieux chêne a vu passer huit ou dix générations de forestiers, un vieil arole 20 générations de bergers!). Un forestier ne vit jamais assez longtemps pour exploiter l'arbre qu'il a planté ou soigné!

- tenter de se représenter les conséquences de ses actes dans l'avenir (réactions de la forêt après une éclaircie, choix des essences)

Dans la société de consommation: la satisfaction immédiate des besoins, la primauté de la nouveauté, des modes, l'«individualisme» sont autant d'hypothèques pour les générations futures.

\section{Accorder une valeur en soi à la nature}

- ne pas couper un arbre parce qu'il est vieux, chargé d'histoire

- traiter une lisière de façon à garder sa diversité faunistique et floristique

- conserver des habitats (qui gênent l'exploitation du bois) ou des biotopes non rentables matériellement.

Dans la société de consommation: la priorité est accordée aux besoins (et aux faux besoins) humains, la nature est au service de l'homo sapiens, sa valeur est mesurée en services matériels rendus.

\section{Utiliser les forces et les cycles de la nature}

- accepter la fertilité naturelle du sol forestier en renonçant aux engrais

- accepter le niveau de résistance naturelle en renonçant aux pesticides.

Dans la société de consommation: on pousse artificiellement la production et la consommation agricole ou industrielle, on utilise massivement des médicaments et des pesticides.

\section{Respecter les rythmes de la nature}

- favoriser le rajeunissement naturel et attendre qu'il s'installe (cela peut durer des décennies)

- abattre les arbres l'hiver pour diminuer les dégâts aux écorces des arbres restants.

Dans la société de consommation: on accélère les rythmes de production et de consommation, nivelle les rythmes propres aux saisons (les fraises à Noël).

\section{Minimaliser les risques encourus}

- répartir les risques de maladies sur plusieurs essences (forêt mélangée si cela correspond aux données écologiques)

- utiliser des essences locales

- exiger la réduction de la pollution de l'air.

Dans la société de consommation: on maximise si possible les bénéfices à court terme au mépris des risques encourus (énergie nucléaire, énergies fossiles, pollution des sols).

\section{Rechercher un mariage durable entre économie} et écologie

- loi du développement durable: exploiter le bois tout en assurant un approvisionnement à long terme (ne pas entamer le capital)

- accepter des défrichements légaux tout en conservant la surface totale de forêt (compensation)

- veiller à conserver un cycle équilibré des matières nutritives en forêt

- couper les arbres atteints du bostryche en cas de nécessité, installer des arbres-pièges (ne pas chercher à éradiquer ces insectes par des traitements chimiques).

Dans la société de consommation: le capital des ressources naturelles est, selon les pays, largement entamé.

\section{Optimiser des choix en considérant la diversité des fonctions}

- faire la part entre l'effet protecteur et économique (au siècle dernier encore, on a rasé des pans de montagne au mépris des effets d'érosion et de la perte des effets protecteurs)

- exploitation minimale ou nulle dans une zone de détente

- garder des îlots de vieux bois (protection des espèces animales).

Société de consommation: la croissance illimitée du produit national brut semble servir encore de modèle à la majorité des décideurs de l'économie.

\section{Pour conclure}

La forêt est à la fois nature et entreprise. Elle offre la possibilité aux éducateurs d'y rencontrer tous les éléments qui permettent d'élaborer une relation de respect avec le paysage naturel ou civilisé. La forêt offre en particulier des exemples positifs de gestion durable des ressources naturelles. Mais pour que les jeunes générations, par exemple les écoliers, puissent vraiment faire cette démarche, non seulement cognitive, mais aussi affective ou pratique, il faut ouvrir la formation et le perfectionnement des enseignants. Il faudrait en particulier tenir davantage compte de la complexité des milieux à découvrir et propager des méthodes pédagogiques adéquates. C'est une des tâches que s'est fixée le programme Découvrir la forêt - CH Waldwochen - Vivere il bosco.

\section{Notes}

' Actuellement, une quinzaine de collaborateurs et collaboratrices réalisent des mandats pour "Découvrir la forêt", pour un volume d'environ cinq postes équivalents de travail. Les projets sont décentralisés, tout en possédant une direction commune. Réunis en équipes linguistiques, ils se concentrent sur le développement des aspects de la pédagogie et de la communication. Les organisations qui soutiennent moralement les projets sont issues aussi bien des milieux natio- 
naux de l'économie et des professions forestières que de la protection de la nature et de l'éducation. Le budget a passé de 20000 francs en 1985, date de la première action, à environ 500000 francs ces dernières années.

${ }^{2}$ Les personnes intéressées peuvent obtenir le détail des questions et les réponses chiffrées chez l'auteur.

\section{Bibliographie}

- Directeurs cantonaux de l'instruction publique: Education à l'environnement dans les écoles publiques, Berne 1988.

- Découvrir la forêt: Projets 1990-1992, Cahier Découvrir la forêt No 2, 1990.
- Lohri Franz: Zwischen Endzeitstimmung und Frühlingserwachen; in: Umweltlernen 59/1991, D-Paderborn.

- Publications didactiques: se renseigner aux adresses cidessous.

\section{Publications didactiques et renseignements}

Suisse romande: Découvrir la forêt, 8, rue des Pêcheurs, 1400 Yverdon-les-Bains, Tél. 042/2102 82

Deutschschweiz: CH Waldwochen, Rebbergstraße, 4800 Zofingen, Tel. 062/510287

Svizzera italiana: Vivere il bosco: Claudia Ribi-Mossi, via FalIeroni 7, 6512 Giubiasco, Tel. 092/276903 